\title{
Was ist Mobbing und welche Formen gibt es?
}

Der Begriff Mobbing wurde in seiner heutigen Bedeutung durch den Arbeitspsychologen und Betriebswirt Heinz Leymann geprägt. Er definierte Mobbing als systematische Schikane am Arbeitsplatz. Von Mobbing wird im vorliegenden Buch gesprochen, wenn systematische Schikanen am Arbeitsplatz bestehen, die zu zunehmender Isolierung unter der Voraussetzung eines Machtungleichgewichtes bei den Betroffenen führen (vgl. Kolodej 2008). Mobbing führt zu einem starken Ungleichgewicht zwischen den Beteiligten. Dieses Ungleichgewicht kann die Anzahl der Personen oder aber ihre Machtbefugnisse betreffen. Das Machtgefälle kann in den Funktionen der Beteiligten begründet sein, indem z. B. ein Vorgesetzter seinen Untergebenen gezielt schikaniert. Es kann jedoch auch eine kleine Gruppe von Personen einer größeren Gruppe gegenüberstehen.

Im Rahmen der Auseinandersetzung mit Mobbing haben sich dementsprechend unterschiedliche Begriffe, die das Machtgefälle zwischen den Beteiligten berücksichtigen, entwickelt. Mobbing wird sowohl als Überbegriff für die systematische Schikane am Arbeitsplatz verwendet als auch für die spezielle Thematik der Schikanen unter hierarchisch gleichgestellten KollegInnen. Der Begriff „Bossing“ hat sich für die Schikane von einem Vorgesetzten zu seinem/n Untergebenen etabliert. „Staffing“ definiert Schikanen von mehreren Untergebenen zu einem Vorgesetzten (vgl. Kolodej 2008). „Bullying“ ist im deutschsprachigen Raum für Mobbing im Schulbereich gebräuchlich. Zudem haben sich in den letzten Jahren die Begriffe Cyber-Mobbing und Cyber-Bullying durchgesetzt, die für die Schikane am Arbeitsplatz und in der Schule mittels der neuen Medien stehen.

C. Kolodej, Psychologische Selbsthilfe bei Mobbing, essentials, https://doi.org/10.1007/978-3-658-19941-8_1 